\title{
Calculation of Dynamic Hardness by Molecular Dynamics
}

\author{
Alexey Bolesta ${ }^{1,2, *}$ \\ ${ }^{1}$ Khristianovich Institute of Theoretical and Applied Mechanics of SB RAS, 630090 Novosibirsk, \\ Russia \\ ${ }^{2}$ Novosibirsk State University, 630090 Novosibirsk, Russia
}

\begin{abstract}
Based on the molecular-dynamic simulation of the impact of a solid ball on the surface of polycrystalline copper, a method for calculating the dynamic hardness of nanocrystalline materials is proposed. It is proposed to carry out the calculation of hardness by dividing the impact work by the squeezed volume. It is shown that this expression of dynamic hardness is consistent with Meyer hardness in the case of quasistatic indentation. As a result of this simulation, it is shown that under conditions when the diameter of the impactor decreases and approaches the crystal lattice constant of the target, the dynamic hardness increases. Also, in the calculations, the impactor density varied approximately twice, which was equal to the density of steel and the density of tungsten carbide. For a striker diameter of $5 \mathrm{~nm}$, dynamic hardness increases with the speed of the striker and does not depend on its density.
\end{abstract}

\section{Introduction}

The concept of dynamic hardness of materials is important when modeling their behavior under shock loading by the methods of continuum mechanics and in experiment. In the present work, based on molecular dynamics simulation of a hard ball impact on the surface of polycrystalline copper, a method for calculating the dynamic hardness of nanocrystalline materials is proposed. It is proposed to carry out the calculation of hardness by dividing the impact work by the displaced volume. This approach is based on early observations that for a rather wide range of experimental conditions, the volume of the imprint formed upon impact of a spherical impactor on the surface of various materials is proportional to its energy [1].

\section{Model}

Modeling was carried out by molecular dynamics (MD). The interaction of copper atoms was calculated using the embedded atom method [2]. The calculation of the motion paths was carried out using the parallel molecular dynamics package LAMMPS [3]. To form the initial data, the atoms were placed in the computational cell in accordance with the face-

\footnotetext{
*Corresponding author: bolesta@itam.nsc.ru
} 
centered cubic lattice. Then, the resulting system was heated above the melting point, maintaining the pressure equal to atmospheric. The obtained melt was sharply cooled at atmospheric pressure to a temperature of 400 to $1200 \mathrm{~K}$, after which, during the evolution of the system at a constant temperature, homogeneous nucleation of copper crystallites occurs in it, which grow for several tens of nanoseconds, forming a polycrystal. Then, to stop grain growth, the system was cooled down to a room temperature of $300 \mathrm{~K}$. Different modes of nucleation and grain growth lead to the formation of a polycrystalline state with different average grain sizes. To identify the local structural state of the atomic system, along with the classical pairwise radial distribution function, we used the analysis of the nearest neighbors [4], which makes it possible to identify atoms with local coordination corresponding to face-centered cubic (FCC), close-packed hexagonal (HCP) and bodycentered cubic ( $\mathrm{BCC}$ ) crystal lattices and disordered phase. To calculate the average grain size in the system, we introduced the concept of FCC + HCP cluster as the set of all bound atoms with FCC and HCP local coordination. The concept of two bound atoms was introduced as a condition that the distance between them at a given moment in time is less than a certain one, calculated as the first minimum in a pair function of radial distribution. As a result of revealing all the FCC + HCP clusters defined in this way, the average number of atoms belonging to them and their average diameter $D$ were calculated based on the assumption of the spherical shape of the clusters, which in the general case is true only with some accuracy.

The impactor was modeled by a solid structureless particle, the interaction of which with copper atoms was described by the repulsive potential (Fig. 1). Such a statement of the problem corresponds to a situation where the hardness of the impactor far exceeds the hardness of the target material, and its deformation can be neglected. The impactor density varied approximately two times and corresponded to the density of steel $\left(7.9 \mathrm{~g} / \mathrm{cm}^{3}\right)$ and the density of tungsten carbide $\left(15.6 \mathrm{~g} / \mathrm{cm}^{3}\right)$. The diameter of the ball ranged from 3 to $5 \mathrm{~nm}$. The grain diameter in polycrystalline copper ranged from 2 to $10 \mathrm{~nm}$. The initial velocity of the striker varied from 300 to $2200 \mathrm{~m} / \mathrm{s}$. Dynamic hardness was calculated as the ratio of the initial kinetic energy of the ball $E_{\mathrm{k}}$ to the squeezed volume on the surface of copper at the moment the projectile stopped $V_{\mathrm{h}}$ :

$$
H_{\mathrm{d}}=E_{\mathrm{k}} / V_{\mathrm{h}}
$$

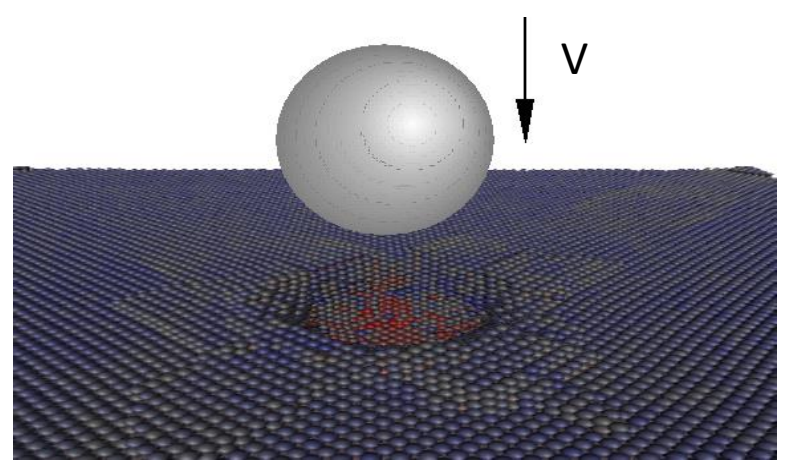

Fig. 1. Molecular dynamics setup. 


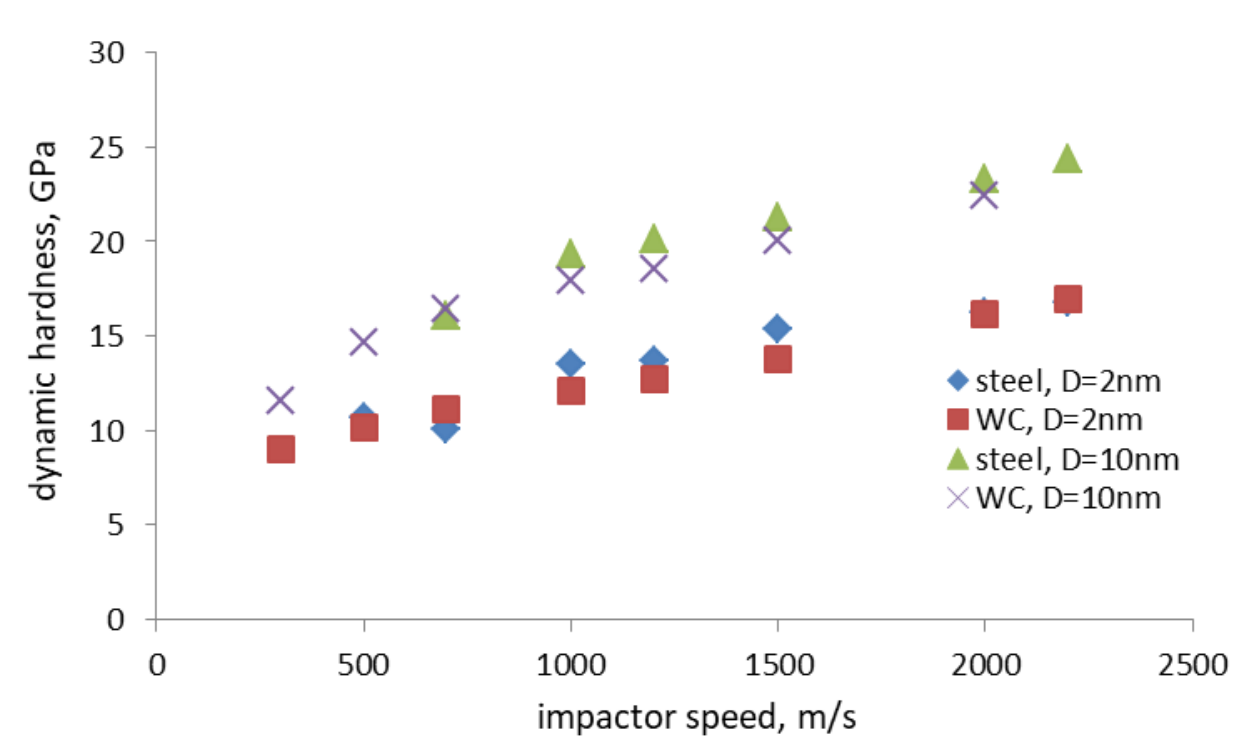

Fig. 2. The dependence of dynamic hardness on the impactor speed for polycrystals of copper with an average grain size $D$ of 2 and $10 \mathrm{~nm}$. The density of the spherical impactor was equal to the density of steel and tungsten carbide.

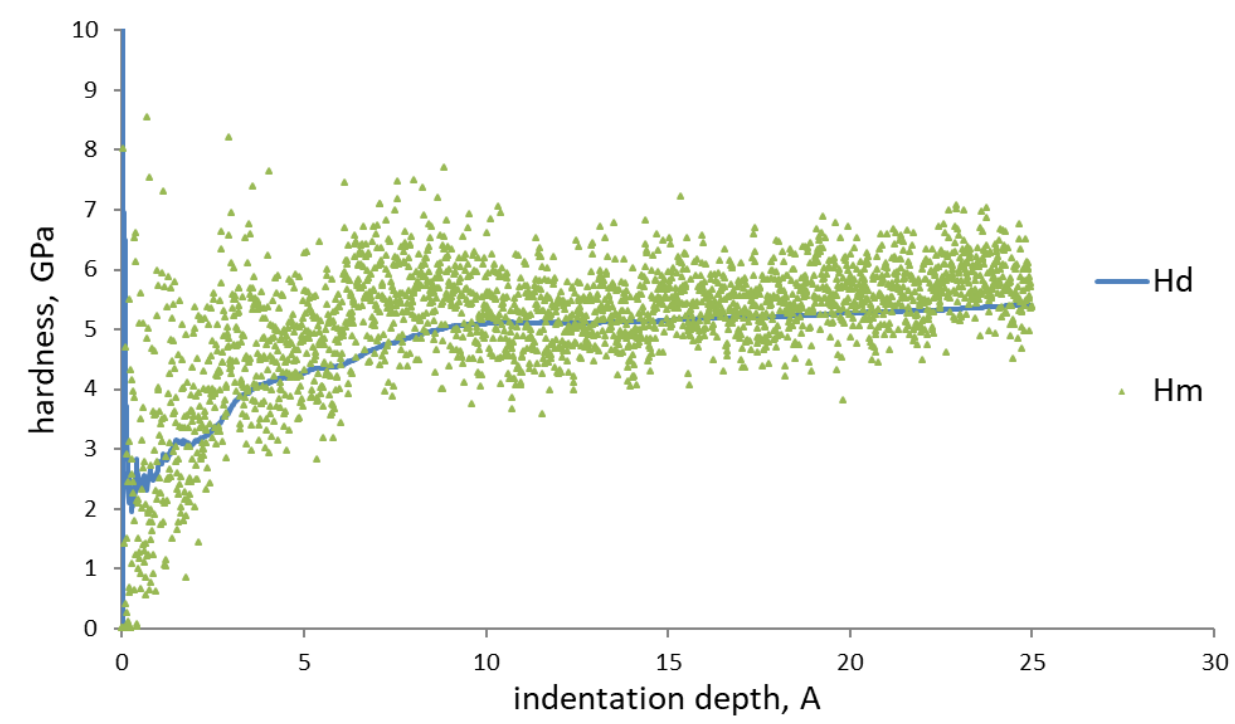

Fig. 3. The dependence of hardness on the indentation depth calculated by dynamic formula (1) - line and by Meyer formula (2) - points. Average grain size $D$ is $2 \mathrm{~nm}$. Diameter of indenter is $5 \mathrm{~nm}$. The speed of indenter is $10 \mathrm{~m} / \mathrm{s}$.

\section{Results}

As a result of the calculations, it was shown that, in contrast to the results described in [1], the dynamic hardness increases with an increase in the impactor speed. This fact is well known for velocities exceeding $1000 \mathrm{~m} / \mathrm{s}$ (see, for example, [5]). An interesting observation 
is a decrease in the calculated dynamic hardness with a diameter of the impactor $D=3 \mathrm{~nm}$, if its density is increased. So with an increase in the mass of the impactor by a factor of two, the hardness increased by a factor of $1.5-2$. At the same time, for the diameter $D=5 \mathrm{~nm}$, the dependence on the density of the impactor practically disappears (Fig. 2). This fact can be qualitatively explained by the inapplicability of the model of continuum mechanics to the impact process for very small sizes of the impactor, comparable to the constant of the crystal lattice, when along with the mechanism of forcing the target there is also a certain "cutting" of its material. But the lack of influence on the calculated hardness of the density of the ball with an increase in its diameter to $5 \mathrm{~nm}$ already shows that this value characterizes only the properties of the target material, and can be confidently presented as a candidate for the role of dynamic hardness.

The results of molecular dynamics calculations of the quasistatic indentation of a spherical indenter at a constant speed of $10 \mathrm{~m} / \mathrm{s}$ also add additional confidence. The statement of the problem in this case is similar to [6] and the result is the good coincidence (Fig. 3) of the behaviour according to dynamic formula (1) with the well-known Meyer formula for static hardness

$$
H_{\mathrm{m}}=F / S \text {, }
$$

considering hardness as a ratio of the indentation force $F$ to the projection area of the imprint $S$. In this case, indentation is performed in a material whose stress-strain diagram is close to an ideal elastic-plastic body [6].

In addition, it should be noted that the scale effect, which was previously described in [7] for quasistatic indentation, is also observed in this work. With a decrease in the size of the impactor, all other conditions of the process being equal, the calculated hardness increases, which can be qualitatively explained by a decrease in the available volume for the generation of defects in the crystal lattice necessary for plastic deformation of the material, despite the fact that the defect at the microscale level itself has a finite volume.

The research was carried out within the framework of the Program of Fundamental Scientific Research of the state academies of sciences in 2013-2020 (project No. AAAA-A17-117030610134-9).

\section{References}

1. D. Tabor, The Hardness of Metals (Oxford Univ. Press, London, 1951)

2. Y. Mishin, M.J. Mehl, D.A. Papaconstantopoulos, A.F. Voter, J.D. Kress, Phys. Rev. B, 63224106 (2001)

3. S.J. Plimpton, J. Comp. Phys. 117, 1-19 (1995)

4. H. Tsuzuki, P.S. Branicio, J.P. Rino, Comput. Phys. Comm. 177, 518-523 (2007)

5. B.J. Koeppel, G. Subhash, Wear 224, 56-67 (1999)

6. A.V. Bolesta, V.M. Fomin, Journal of Applied Mechanics and Technical Physics 55, 800-811 (2014)

7. A.V. Bolesta, V.M. Fomin, Physical Mesomechanics 12, 3-4, 117-123 (2009) 